\title{
Best proximity point theorems for probabilistic proximal cyclic contraction with applications in nonlinear programming
}

Reza Saadati

"Correspondence: rsaadati@eml.cc Department of Mathematics, Iran University of Science and Technology, Narmak, Tehran, Iran

\begin{abstract}
In this paper, we derive a best proximity point theorem for non-self-mappings satisfied proximal cyclic contraction in PM-spaces and this shows the existence of optimal approximate solutions of certain simultaneous fixed point equations in the event that there is no solution. As an application we consider a nonlinear programming problem. Our results extend and improve the recent results of (Sadiq Basha in Nonlinear Anal. 74(17):5844-5850, 2011).
\end{abstract}

Keywords: optimal approximate solution; fixed point; best proximity point; proximal contraction; proximal cyclic contraction

\section{Introduction}

Best proximity point theorems are those results that provide sufficient conditions for the existence of a best proximity point and algorithms for finding best proximity points. It is interesting to note that best proximity point theorems generalized fixed point theorems in a natural fashion. Indeed, if the mapping under consideration is a self-mapping, a best proximity point becomes a fixed point.

One of the most interesting is the study of the extension of Banach contraction principle to the case of non-self-mappings. In fact, given nonempty closed subsets $A$ and $B$ of a complete PM-space $(X, F, *)$, a contraction non-self-mapping $T: A \rightarrow B$ does not necessarily has a fixed point. Eventually, it is quite natural to find an element $x$ such that $F_{x, T x}(t)$ is maximum for a given problem which implies that $x$ and $T x$ are in close proximity to each other.

Many problems can be formulated as equations of the form $T x=x$, where $T$ is a selfmapping in some suitable framework. Fixed point theory finds the existence of a solution to such generic equations and brings out the iterative algorithms to compute a solution to such equations.

However, in the case that $T$ is non-self-mapping, the aforementioned equation does not necessarily have a solution. In such a case, it is worthy to determine an approximate solution $x$ such that the error $F_{x, T x}(t)$ is maximum.

\section{Preliminaries}

Throughout this paper, the space of all probability distribution functions (briefly, d.f.'s) is denoted by $\Delta^{+}=\{F: \mathbf{R} \cup\{-\infty,+\infty\} \rightarrow[0,1]: F$ is left-continuous and non-decreasing on

(c) 2015 Saadati. This article is distributed under the terms of the Creative Commons Attribution 4.0 International License (http://creativecommons.org/licenses/by/4.0/), which permits unrestricted use, distribution, and reproduction in any medium, provided you give appropriate credit to the original author(s) and the source, provide a link to the Creative Commons license, and indicate if changes were made. 
$\mathbf{R}, F(0)=0$, and $F(+\infty)=1\}$ and the subset $D^{+} \subseteq \Delta^{+}$is the set $D^{+}=\left\{F \in \Delta^{+}: l^{-} F(+\infty)=1\right\}$. Here $l^{-} f(x)$ denotes the left limit of the function $f$ at the point $x, l^{-} f(x)=\lim _{t \rightarrow x^{-}} f(t)$. The space $\Delta^{+}$is partially ordered by the usual point-wise ordering of functions, i.e., $F \leq G$ if and only if $F(t) \leq G(t)$ for all $t$ in $\mathbf{R}$. The maximal element for $\Delta^{+}$in this order is the d.f. given by

$$
\varepsilon_{0}(t)= \begin{cases}0, & t \leq 0 \\ 1, & t>0\end{cases}
$$

Definition $2.1([1])$ A mapping $*:[0,1] \times[0,1] \rightarrow[0,1]$ is a continuous $t$-norm if $*$ satisfies the following conditions:

(a) $*$ is commutative and associative;

(b) $*$ is continuous;

(c) $a * 1=a$ for all $a \in[0,1]$;

(d) $a * b \leq c * d$ whenever $a \leq c$ and $b \leq d$, and $a, b, c, d \in[0,1]$.

Two typical examples of a continuous $t$-norm are $a * b=a b$ and $a * b=\min (a, b)$.

A $t$-norm $*$ is said to be of Hadžić type if

$$
\forall \epsilon \in(0,1) \exists \delta \in(0,1): \quad a>1-\delta \Rightarrow \overbrace{a * a * \cdots * a}^{n}>1-\epsilon \quad(n \geq 1) .
$$

The $t$-norm minimum is a trivial example of a $t$-norm of Hadžić type, but there exists a $t$-norm of Hadžić type weaker than minimum (see [2]).

Definition 2.2 A probabilistic metric space (briefly, PM-space) is a triple $(X, F, *)$, where $X$ is a nonempty set, $*$ is a continuous $t$-norm, and $F$ is a mapping from $X \times X$ into $D^{+}$ such that, if $F_{x, y}$ denotes the value of $F$ at the pair $(x, y)$, the following conditions hold: for all $x, y, z$ in $X$,

(PM1) $F_{x, y}(t)=\varepsilon_{0}(t)$ for all $t>0$ if and only if $x=y$;

(PM2) $F_{x, y}(t)=F_{y, x}(t)$

(PM3) $F_{x, z}(t+s) \geq F_{x, y}(t) * F_{y, z}(s)$ for all $x, y, z \in X$ and $t, s \geq 0$.

For more details and examples of these spaces see also [3-5].

Definition 2.3 Let $(X, F, *)$ be a PM-space.

(1) A sequence $\left\{x_{n}\right\}_{n}$ in $X$ is said to be convergent to $x$ in $X$ if, for every $\epsilon>0$ and $\lambda>0$, there exists a positive integer $N$ such that $F_{x_{n}, x}(\epsilon)>1-\lambda$ whenever $n \geq N$.

(2) A sequence $\left\{x_{n}\right\}_{n}$ in $X$ is called Cauchy sequence if, for every $\epsilon>0$ and $\lambda>0$, there exists a positive integer $N$ such that $F_{x_{n}, x_{m}}(\epsilon)>1-\lambda$ whenever $n, m \geq N$.

(3) A PM-space $(X, F, *)$ is said to be complete if and only if every Cauchy sequence in $X$ is convergent to a point in $X$.

Definition 2.4 Let $(X, F, *)$ be a PM-space. For each $p$ in $X$ and $\lambda>0$, the strong $\lambda$-neighborhood of $p$ is the set

$$
N_{p}(\lambda)=\left\{q \in X: F_{p, q}(\lambda)>1-\lambda\right\}
$$


and the strong neighborhood system for $X$ is the union $\bigcup_{p \in V} \mathcal{N}_{p}$ where $\mathcal{N}_{p}=\left\{N_{p}(\lambda)\right.$ : $\lambda>0\}$.

The strong neighborhood system for $X$ determines a Hausdorff topology for $X$.

Theorem 2.5 ([1]) If $(X, F, *)$ is a PM-space and $\left\{p_{n}\right\}$ and $\left\{q_{n}\right\}$ are sequences such that $p_{n} \rightarrow p$ and $q_{n} \rightarrow q$, then $\lim _{n \rightarrow \infty} F_{p_{n}, q_{n}}(t)=F_{p, q}(t)$ for every continuity point $t$ of $F_{p, q}$.

Lemma 2.6 ([2]) Let $(X, F, *)$ be a Menger PM-space with $*$ of Hadžić-type and $\left\{x_{n}\right\}$ be a sequence in $X$ such that, for some $k \in(0,1)$,

$$
F_{x_{n}, x_{n+1}}(k t) \geq F_{x_{n-1}, x_{n}}(t) \quad(n \geq 1, t>0) .
$$

Then $\left\{x_{n}\right\}$ is a Cauchy sequence.

Let $A$ and $B$ be two nonempty subsets of a PM-space and $t>0$, the following notions and notations are used in the sequel.

$$
\begin{aligned}
& F_{A, B}(t):=\sup \left\{F_{x, y}(t): x \in A, y \in B\right\}, \\
& A_{0}:=\left\{x \in A: F_{x, y}(t)=F_{A, B}(t) \text { for some } y \in B\right\}, \\
& B_{0}:=\left\{y \in B: F_{x, y}(t)=F_{A, B}(t) \text { for some } x \in A\right\} .
\end{aligned}
$$

Definition 2.7 Let $(X, F, *)$ be a PM-space. Given non-self-mappings $S: A \rightarrow B$ and $T$ : $B \rightarrow A$, the pair $(S, T)$ is said to form a proximal cyclic contraction if there exists a nonnegative number $\alpha<1$ such that

$$
\left.\begin{array}{l}
F_{u, S x}(t)=F_{A, B}(t), \\
F_{v, T y}(t)=F_{A, B}(t)
\end{array}\right\} \quad \Longrightarrow \quad F_{u, v}(t) \geq \min \left\{F_{x, y}\left(\frac{t}{\alpha}\right), F_{A, B}(t)\right\}
$$

for all $u, x$ in $A$ and $v, y$ in $B$ and $t>0$.

Note that, if $S$ is a self-mapping that is a contraction, then the pair the pair $(S, S)$ forms a proximal cyclic contraction.

Definition 2.8 Let $(X, F, *)$ be a PM-space. A mapping $S: A \rightarrow B$ is said to be a proximal contraction of the first kind if there exists a non-negative number $\alpha<1$ such that

$$
\left.\begin{array}{l}
F_{u_{1}, S x_{1}}(t)=F_{A, B}(t), \\
F_{u_{2}, S x_{2}}(t)=F_{A, B}(t)
\end{array}\right\} \quad \Longrightarrow \quad F_{u_{1}, u_{2}}(\alpha t) \geq F_{x_{1}, x_{2}}(t)
$$

for all $u_{1}, u_{2}, x_{1}, x_{2}$ in $A$ and $t>0$.

Definition 2.9 Let $(X, F, *)$ be a PM-space. A mapping $S: A \rightarrow B$ is said to be a proximal contraction of the second kind if there exists a non-negative number $\alpha<1$ such that

$$
\left.\begin{array}{l}
F_{u_{1}, S x_{1}}(t)=F_{A, B}(t), \\
F_{u_{2}, S x_{2}}(t)=F_{A, B}(t)
\end{array}\right\} \quad \Longrightarrow \quad F_{S u_{1}, S u_{2}}(\alpha t) \geq F_{S x_{1}, S x_{2}}(t)
$$

for all $u_{1}, u_{2}, x_{1}, x_{2}$ in $A$ and $t>0$. 
Definition 2.10 Let $(X, F, *)$ be a PM-space. Given a mapping $S: A \rightarrow B$ and an isometry $g: A \rightarrow A$, the mapping $S$ is said to preserve isometric distance with respect to $g$ if

$$
F_{S g x_{1}, S g x_{2}}(t)=F_{S x_{1}, S x_{2}}(t)
$$

for all $x_{1}$ and $x_{2}$ in $A$. and $t>0$.

Definition 2.11 Let $(X, F, *)$ be a PM-space. An element $x$ in $A$ is said to be a best proximity point of the mapping $S: A \rightarrow B$ if it satisfies the condition that

$$
F_{x, S x}(t)=F_{A, B}(t)
$$

for all $x$ in $A$ and $t>0$.

It can be observed that a best proximity reduces to a fixed point if the underlying mapping is a self-mapping.

Definition 2.12 Let $(X, F, *)$ be a PM-space. $B$ is said to be approximatively compact with respect to $A$ if every sequence $\left\{y_{n}\right\}$ of $B$ satisfying the condition that for all $t>0, F_{x, y_{n}}(t) \rightarrow$ $F_{x, B}(t)$ for some $x$ in $A$ has a convergent subsequence.

It is easy to observe that every set is approximatively compact with respect to itself, and that every compact set is approximatively compact. Moreover, $A_{0}$ and $B_{0}$ are non-void if $A$ is compact and $B$ is approximatively compact with respect to $A$.

\section{Proximal contractions}

The following main result is a generalized best proximity point theorem for non-self proximal contractions of the first kind. Our results extend and improve some results of [6].

Theorem 3.1 Let $A$ and $B$ be non-void closed subsets of a complete PM-space $(X, F, *)$ with $*$ of Hadžić-type such that $A_{0}$ and $B_{0}$ are non-void. Let $S: A \rightarrow B, T: B \rightarrow A$ and $g: A \cup B \rightarrow A \cup B$ satisfy the following conditions:

(a) $S$ and $T$ are proximal contractions of the first kind.

(b) $S\left(A_{0}\right) \subseteq B_{0}$ and $T\left(B_{0}\right) \subseteq A_{0}$.

(c) The pair $(S, T)$ forms a proximal cyclic contraction.

(d) $g$ is an isometry.

(e) $A_{0} \subseteq g\left(A_{0}\right)$ and $B_{0} \subseteq g\left(B_{0}\right)$.

Then there exist a unique element $x$ in $A$ and a unique element $y$ in $B$ satisfying the conditions that

$$
\begin{aligned}
& F_{g x, S x}(t)=F_{A, B}(t), \\
& F_{g y, T y}(t)=F_{A, B}(t), \\
& F_{x, y}(t)=F_{A, B}(t) .
\end{aligned}
$$

Further, for any fixed element $x_{0}$ in $A_{0}$, the sequence $\left\{x_{n}\right\}$, defined by

$$
F_{g x_{n+1}, S x_{n}}(t)=F_{A, B}(t)
$$


converges to the element $x$. For any fixed element $y_{0}$ in $B_{0}$, the sequence $\left\{y_{n}\right\}$, defined by

$$
F_{g y_{n+1}, T y_{n}}(t)=F_{A, B}(t)
$$

converges to the element $y$.

On the other hand, a sequence $\left\{u_{n}\right\}$ of elements in A converges to $x$ if there is a sequence $\left\{\epsilon_{n}\right\}$ of positive numbers for which

$$
\lim _{n \rightarrow \infty} \prod_{i=0}^{n}\left(1-\epsilon_{i}\right)=1
$$

in which

$$
\prod_{i=0}^{n} a_{i}=a_{0} * \cdots * a_{n}
$$

for $a_{i} \in(0,1]$ and

$$
F_{u_{n+1}, z_{n+1}}(t) \geq 1-\epsilon_{n}
$$

where $z_{n+1} \in A$ satisfies the condition that

$$
F_{z_{n+1}, S u_{n}}(t)=F_{A, B}(t)
$$

for $t>0$.

Proof Let $x_{0}$ be an element in $A_{0}$. In view of the facts that $S\left(A_{0}\right)$ is contained in $B_{0}$ and that $A_{0}$ is contained in $g\left(A_{0}\right)$, it is ascertained that there is an element $x_{1}$ in $A_{0}$ such that

$$
F_{g x_{1}, S x_{0}}(t)=F_{A, B}(t)
$$

for $t>0$. Again, since $S\left(A_{0}\right)$ is contained in $B_{0}$, and $A_{0}$ is contained in $g\left(A_{0}\right)$, there exists an element $x_{2}$ in $A_{0}$ such that

$$
F_{g x_{2}, S x_{1}}(t)=F_{A, B}(t)
$$

for $t>0$. One can proceed further in a similar fashion to find $x_{n}$ in $A_{0}$. Having chosen $x_{n}$, one can determine an element $x_{n+1}$ in $A_{0}$ such that

$$
F_{g x_{n+1}, S x_{n}}(t)=F_{A, B}(t)
$$

because of the facts that $S\left(A_{0}\right)$ is contained in $B_{0}$ and that $A_{0}$ is contained in $g\left(A_{0}\right)$. In light of the facts that $g$ is an isometry and that $S$ is a proximal contraction of the first kind,

$$
F_{x_{n}, x_{n+1}}(\alpha t)=F_{g x_{n}, g x_{n+1}}(\alpha t) \geq F_{x_{n-1}, x_{n}}(t)
$$

for $t>0$. Therefore, by Lemma 2.6, $\left\{x_{n}\right\}$ is a Cauchy sequence and hence converges to some element $x$ in $A$. Similarly, in view of the facts that $T\left(B_{0}\right)$ is contained in $A_{0}$ and that 
$B_{0}$ is contained in $g\left(B_{0}\right)$, it is guaranteed that there is a sequence $\left\{y_{n}\right\}$ of elements in $B_{0}$ such that

$$
F_{g y_{n+1}, T y_{n}}(t)=F_{A, B}(t)
$$

for $t>0$. Because $g$ is an isometry and $T$ is a proximal contraction of the first kind, it follows that

$$
F_{y_{n}, y_{n+1}}(\alpha t)=F_{g y_{n}, g y_{n+1}}(\alpha t) \geq F_{y_{n-1}, y_{n}}(t)
$$

for $t>0$. Therefore, by Lemma 2.6, $\left\{y_{n}\right\}$ is a Cauchy sequence and hence converges to some element $y$ in $B$. Since the pair $(S, T)$ forms a proximal cyclic contraction and $g$ is an isometry, it follows that

$$
F_{x_{n+1}, y_{n+1}}(t)=F_{g x_{n+1}, g y_{n+1}}(t) \geq \min \left\{F_{x_{n}, y_{n}}\left(\frac{t}{\alpha}\right), F_{A, B}(t)\right\}
$$

for $t>0$.

Letting $n \rightarrow \infty$, since $F_{x, y}(t) \leq F_{x, y}(t / \alpha)$ we have,

$$
F_{x, y}(t)=F_{A, B}(t)
$$

for $t>0$. Thus, it can be concluded that $x$ is a member of $A_{0}$ and that $y$ is a member of $B_{0}$. Since $S\left(A_{0}\right)$ is contained in $B_{0}$, and $T\left(B_{0}\right)$ is contained in $A_{0}$, there exist an element $u$ in $A$ and an element $v$ in $B$ such that

$$
\begin{aligned}
& F_{u, S x}(t)=F_{A, B}(t), \\
& F_{v, T y}(t)=F_{A, B}(t)
\end{aligned}
$$

for $t>0$. Because $S$ is a proximal contraction of the first kind,

$$
F_{u, g x_{n+1}}(\alpha t) \geq F_{x, x_{n}}(t)
$$

for $t>0$. Letting $n \rightarrow \infty$, we have the result that $u=g x$. Thus, it follows that

$$
F_{g x, S x}(t)=F_{A, B}(t)
$$

for $t>0$. Similarly, it can be shown that $v=g y$ and hence

$$
F_{g y, T y}(t)=F_{A, B}(t)
$$

for $t>0$. To prove the uniqueness, let us suppose that there exist elements $x^{*}$ in $A$ and $y^{*}$ in $B$ such that

$$
\begin{aligned}
& F_{g x^{*}, S x^{*}}(t)=F_{A, B}(t), \\
& F_{g y^{*}, T y^{*}}(t)=F_{A, B}(t)
\end{aligned}
$$


for $t>0$. Since $g$ is an isometry, and the non-self-mappings $S$ and $T$ are proximal contractions of the first kind, it follows that

$$
\begin{aligned}
& F_{x, x^{*}}(\alpha t)=F_{g x, g x^{*}}(\alpha t) \geq F_{x, x^{*}}(t), \\
& F_{y, y^{*}}(\alpha t)=F_{g y, g y^{*}}(\alpha t) \geq F_{y, y^{*}}(t)
\end{aligned}
$$

for $t>0$. Therefore, $x$ and $x^{*}$ are identical, and $y$ and $y^{*}$ are identical.

On the other hand, let $\left\{u_{n}\right\}_{n=0}^{\infty}$ in which $u_{0}=x_{0}$ be a sequence of elements in $A$ and $\left\{\epsilon_{n}\right\}$ a sequence $(0,1)$ such that

$$
\lim _{n \rightarrow \infty} \prod_{i=0}^{n}\left(1-\epsilon_{i}\right)=1
$$

and

$$
F_{u_{n+1}, z_{n+1}}(t) \geq 1-\epsilon_{n}
$$

where $z_{n+1} \in A$ satisfies the condition that

$$
F_{z_{n+1}, S u_{n}}(t)=F_{A, B}(t)
$$

for $t>0$. Since $S$ is a proximal contraction of the first kind,

$$
F_{x_{n+1}, z_{n+1}}(\alpha t) \geq F_{x_{n}, u_{n}}(t) .
$$

Given $\delta \in(0,1)$, for all $n \geq N$ we have

$$
\begin{aligned}
F_{x_{n+1}, u_{n+1}}(t+\delta) & \geq F_{x_{n+1}, z_{n+1}}(t) * F_{z_{n+1}, u_{n+1}}(\delta) \\
& \geq F_{x_{n}, u_{n}}\left(\frac{t}{\alpha}\right) *\left(1-\epsilon_{n}\right) \\
& \geq F_{x_{n}, u_{n}}\left(\frac{t}{\alpha^{2}}\right) *\left(1-\epsilon_{n-1}\right) *\left(1-\epsilon_{n}\right) \\
& \geq \cdots \geq F_{x_{0}, u_{0}}\left(\frac{t}{\alpha^{n+1}}\right) * \prod_{i=0}^{n}\left(1-\epsilon_{i}\right)
\end{aligned}
$$

for $t>0$. Since $\delta \in(0,1)$ was arbitrary, we have

$$
F_{x_{n+1}, u_{n+1}}(t) \geq \prod_{i=0}^{n}\left(1-\epsilon_{i}\right)
$$

for $t>0$. Now,

$$
\begin{aligned}
F_{u_{n+1}, x}(2 t) & \geq F_{u_{n+1}, x_{n+1}}(t) * F_{x_{n+1}, x}(t) \\
& \geq \prod_{i=0}^{n}\left(1-\epsilon_{i}\right) * F_{x_{n+1}, x}(t)
\end{aligned}
$$


for $t>0$. Then

$$
\lim _{n \rightarrow \infty} F_{u_{n+1}, x}(2 t) \rightarrow 1
$$

for $t>0$, and it can be concluded that $\left\{u_{n}\right\}$ converges to $x$. This completes the proof of the theorem.

The following example illustrates the preceding generalized best proximity point theorem.

Example 3.2 Consider the complete PM-space $(\mathbf{R}, F$, min) where

$$
F_{x, y}(t)=\frac{t}{t+|x-y|},
$$

when $t>0$ and

$$
F_{x, y}(t)=0,
$$

when $t \leq 0$ for $x, y$ in $\mathbf{R}$.

Let $A=[-1,0]$ and $B=[0,1]$.

Let $S: A \rightarrow B, T: B \rightarrow A$, and $g: A \cup B \rightarrow A \cup B$ be defined as

$$
\begin{aligned}
& S(x)=\frac{-x}{2}, \\
& T(y)=\frac{-y}{2}, \\
& g(x)=-x .
\end{aligned}
$$

Then it is easy to see that

$$
F_{A, B}(t)=1,
$$

$A_{0}=\{0\}$ and $B_{0}=\{0\}$. The mapping $g$ is an isometry and the non-self-mappings $S$ and $T$ are proximal contractions of the first kind, and the pair $(S, T)$ forms a proximal cyclic contraction. The other hypotheses of Theorem 3.1 are also satisfied. Further, it is easy to observe that the element 0 in $A$ and $B$ satisfy the conditions in the conclusion of the preceding result.

If $g$ is assumed to be the identity mapping, then Theorem 3.1 yields the following best proximity point result.

Corollary 3.3 Let $A$ and $B$ be non-void closed subsets of a complete PM-space $(X, F, *)$ with * of Hadžić-type such that $A_{0}$ and $B_{0}$ are non-void. Let $S: A \rightarrow B$ and $T: B \rightarrow A$ satisfy the following conditions:

(a) $S$ and $T$ are proximal contractions of the first kind.

(b) $S\left(A_{0}\right) \subseteq B_{0}$ and $T\left(B_{0}\right) \subseteq A_{0}$.

(c) The pair $(S, T)$ forms a proximal cyclic contraction. 
Then there exist a unique element $x$ in $A$ and a unique element $y$ in $B$ satisfying the conditions that

$$
\begin{aligned}
& F_{x, S x}(t)=F_{A, B}(t), \\
& F_{y, T y}(t)=F_{A, B}(t), \\
& F_{x, y}(t)=F_{A, B}(t)
\end{aligned}
$$

for $t>0$.

Theorem 3.4 Let $A$ and $B$ be non-void closed subsets of a complete PM-space $(X, F, *)$ with $*$ of Hadžić-type such that $A_{0}$ and $B_{0}$ are non-void. Let $S: A \rightarrow B$ and $g: A \rightarrow A$ satisfy the following conditions:

(a) $S$ is a proximal contraction of the first and second kind.

(b) $S\left(A_{0}\right)$ is contained in $B_{0}$.

(c) $g$ is an isometry.

(d) S preserves isometric distance with respect to $g$.

(e) $A_{0}$ is contained in $g\left(A_{0}\right)$.

Then there exists a unique element $x$ in $A$ such that

$$
F_{g x, S x}(t)=F_{A, B}(t)
$$

for $t>0$. Further, for any fixed element $x_{0}$ in $A_{0}$, the sequence $\left\{x_{n}\right\}$, defined by

$$
F_{g x_{n+1}, S x_{n}}(t)=F_{A, B}(t)
$$

converges to the element $x$ for $t>0$.

On the other hand, a sequence $\left\{u_{n}\right\}$ of elements in A converges to $x$ if there is a sequence $\left\{\epsilon_{n}\right\}$ of positive numbers for which

$$
\lim _{n \rightarrow \infty} \prod_{i=0}^{n}\left(1-\epsilon_{i}\right)=1
$$

and

$$
F_{u_{n+1}, z_{n+1}}(t) \geq 1-\epsilon_{n},
$$

where $z_{n+1} \in A$ satisfies the condition that

$$
F_{z_{n+1}, S u_{n}}(t)=F_{A, B}(t)
$$

for $t>0$.

Proof Proceeding as in Theorem 3.1, it is possible to find a sequence $\left\{x_{n}\right\}$ of elements in $A_{0}$ such that

$$
F_{g x_{n+1}, S x_{n}}(t)=F_{A, B}(t)
$$


for $t>0$ and for all non-negative integral values of $n$, because of the facts that $S\left(A_{0}\right)$ is contained in $B_{0}$ and that $A_{0}$ is contained in $g\left(A_{0}\right)$. Due to the facts that $S$ is a proximal contraction of the first kind and $g$ is an isometry,

$$
F_{x_{n}, x_{n+1}}(\alpha t)=F_{g x_{n}, g x_{n+1}}(\alpha t) \geq F_{x_{n-1}, x_{n}}(t)
$$

for $t>0$. Therefore, by Lemma 2.6, $\left\{x_{n}\right\}$ is a Cauchy sequence and hence converges to some element $x$ in $A$. Because of the facts that $S$ is a proximal contraction of the second kind and preserves the isometric distance with respect to $g$,

$$
F_{S x_{n}, S x_{n+1}}(\alpha t)=F_{S g x_{n}, S g x_{n+1}}(\alpha t) \geq F_{S x_{n-1}, S x_{n}}(t)
$$

for $t>0$. Therefore, by Lemma 2.6, $\left\{S x_{n}\right\}$ is a Cauchy sequence and hence converges to some element $y$ in $B$. Thus, it can be concluded that

$$
F_{g x, y}(t)=\lim _{n \rightarrow \infty} F_{g x_{n+1}, S x_{n}}(t)=F_{A, B}(t) .
$$

Eventually, $g x$ is an element of $A_{0}$. Because of the fact that $A_{0}$ is contained in $g\left(A_{0}\right), g x=g z$ for some member $z$ in $A_{0}$. Owing to the fact that $g$ is an isometry, $F_{x, z}(t)=F_{g x, g z}(t)=1$. Consequently, the elements $x$ and $z$ must be identical, and hence $x$ becomes an element of $A_{0}$. Because $S\left(A_{0}\right)$ is contained $B_{0}$,

$$
F_{u, S x}(t)=F_{A, B}(t)
$$

for $t>0$, for some element $u$ in $A$. On account of the fact that the mapping $S$ is a proximal contraction of the first kind,

$$
F_{u, g x_{n+1}}(\alpha t) \geq F_{x, x_{n}}(t)
$$

for $t>0$. As a result, the sequence $\left\{g\left(x_{n}\right)\right\}$ must converge to $u$. However, because of the continuity of $g$, the sequence $\left\{g\left(x_{n}\right)\right\}$ converges to $g x$ as well. Therefore, $u$ and $g x$ must be identical. Thus, we have the result that

$$
F_{g x, S x}(t)=F_{z, S x}(t)=F_{A, B}(t)
$$

for $t>0$. The uniqueness and the remaining part of the proof follow as in Theorem 3.1. This completes the proof of the theorem.

The preceding generalized best proximity point theorem is illustrated by the following example.

Example 3.5 Consider the complete PM-space (R, $F$, min) where

$$
F_{x, y}(t)=\frac{t}{t+|x-y|},
$$

when $t>0$, and

$$
F_{x, y}(t)=0,
$$

when $t \leq 0$ for $x, y \in \mathbf{R}$. 
Let $A=[-1,1]$ and $B=[-3,-2] \cup[2,3]$. Then $F_{A, B}(t)=\frac{t}{t+1}, A_{0}=\{-1,1\}$, and $B_{0}=\{-2,2\}$. Let $S: A \rightarrow B$ be defined as

$$
S x= \begin{cases}2 & \text { if } x \text { is rational } \\ 3 & \text { otherwise }\end{cases}
$$

Then $S$ is a proximal contraction of the first and second kind, and $S\left(A_{0}\right) \subseteq B_{0}$.

Further, let $g: A \rightarrow A$ be defined as $g x=-x$. Then $g$ is an isometry, $S$ preserves the isometric distance with respect to $g$, and $A_{0} \subseteq g\left(A_{0}\right)$. It can also be observed that $F_{g(-1), S(-1)}(t)=F_{A, B}(t)$ for $t>0$.

If $g$ is assumed to be the identity mapping, then Theorem 3.4 yields the following best proximity point theorem.

Corollary 3.6 Let $A$ and $B$ be non-void closed subsets of a complete PM-space $(X, F, *)$ with $*$ of Hadžić-type such that $A_{0}$ and $B_{0}$ are non-void. Let $S: A \rightarrow B$ satisfy the following conditions:

(a) $S$ is a proximal contraction of the first and second kind.

(b) $S\left(A_{0}\right)$ is contained in $B_{0}$.

Then there exists a unique element $x$ in $A$ such that

$$
F_{x, S x}(t)=F_{A, B}(t)
$$

Further, for any fixed element $x_{0}$ in $A_{0}$, the sequence $\left\{x_{n}\right\}$, defined by

$$
F_{x_{n+1}, S x_{n}}(t)=F_{A, B}(t)
$$

converges to the best proximity point $x$ of $S$.

\section{Application}

A solution to the nonlinear programming problem

$$
\max _{x \in A} F_{x, T x}(t)
$$

is fundamentally an ideal optimal approximate solution to the equation $T x=x$ which is shifting to have a solution when $T$ is supposed to be a non-self-mapping.

Considering the fact that $F_{x, T x}(t)$ is at least $F_{A, B}(t)$ for all $x$ in $A$, a solution $x$ to the aforementioned nonlinear programming problem becomes an approximate solution with the lowest possible error to the corresponding equation $T x=x$ if it satisfies the condition that $F_{x, T x}(t)=F_{A, B}(t)$ for $t>0$. 


\section{Acknowledgements}

The author is thankful to the three anonymous referees for giving valuable comments and suggestions, which helped to improve the final version of this paper.

Received: 31 January 2015 Accepted: 17 May 2015 Published online: 06 June 2015

\section{References}

1. Schweizer, B, Sklar, A: Probabilistic Metric Spaces. North-Holland Series in Probability and Applied Mathematics. North-Holland, New York (1983)

2. Hadžić, O, Pap, E: Fixed Point Theory in Probabilistic Metric Spaces. Mathematics and Its Applications, vol. 536. Kluwer Academic, Dordrecht (2001)

3. Hussain, N, Pathak, HK, Tiwari, S: Application of fixed point theorems to best simultaneous approximation in ordered semi-convex structure. J. Nonlinear Sci. Appl. 5(4), 294-306 (2012) (special issue)

4. Chauhan, S, Pant, BD: Fixed point theorems for compatible and subsequentially continuous mappings in Menger spaces. J. Nonlinear Sci. Appl. 7(2), 78-89 (2014)

5. Miheţ, D: Common coupled fixed point theorems for contractive mappings in fuzzy metric spaces. J. Nonlinear Sci. Appl. 6(1), 35-40 (2013)

6. Sadiq Basha, S: Best proximity point theorems generalizing the contraction principle. Nonlinear Anal. 74(17), 5844-5850 (2011)

\section{Submit your manuscript to a SpringerOpen ${ }^{\circ}$ journal and benefit from:}

- Convenient online submission

Rigorous peer review

- Immediate publication on acceptance

- Open access: articles freely available online

- High visibility within the field

- Retaining the copyright to your article 